\title{
A Study of Background Subtraction Method for Nal(TI) Instrument Spectrum Based on Adaptive FWHM
}

\author{
Jianfeng $\mathrm{He}^{1, \text { a }}$, Hailing Xiao, a , Yaozong Yang ${ }^{2, \mathrm{~b}}$, Jianhui $\mathrm{Qu}^{2, \mathrm{c}}$, \\ Hongkun $X u^{3 . d}$, Liu Lin ${ }^{1 . a}$ \\ ${ }^{1}$ Fundamental Science on Radioactive Geology and Exploration Technology Laboratory, East China \\ Institute of Technology, NanChang 330013, China; \\ ${ }^{2}$ Jiujiang Power Supply Subsidiary Company of State Grid Jianxi Electronic Power Company \\ JiuJiang, 332000, China; \\ ${ }^{3}$ Beijing Research Center for Radiation Application, Beijing 100015, China. \\ ahjf_10@yeah.net, ${ }^{b}$ alayaozong@126.com, ‘ qjh-2001-6@163.com, dxhk_auto@163.com
}

Keywords: Nal(TI) Instrument Spectra, Background Subtraction, Adaptive FWHM, SNIP.

\begin{abstract}
How to effectively subtract the background is one of the key technologies of spectrum date analysis in the gamma-ray energy spectra measurements. The paper, according to the SNIP(Statistics Sensitive Nonlinear Iterative Peak-Clipping Algorithm) method that has many advantages on background subtraction in the full spectrum, elaborates the principles of improved SNIP algorithm and the process of background subtraction in detail. However, it is because of traditional SNIP algorithm when deducting the background that the parameter $m$ value of transform window-width is difficult to be determined automatically, by analyzing characteristics of $\mathrm{NaI}(\mathrm{Tl})$ scintillation detector and the physical characteristics of forming spectra, the method for deducting the background based on SNIP of adaptive FWHM(The Full Width of the peak at Half Maximum) is proposed, which is used to solve the problems effectively on valuing the parameter m both automatically and accurately. Multiple sets of spectral data show that the improved SNIP method can correctly subtract the contributions of Compton scattering effect to spectra peak counts, weakens background counting of scattering peak, is simple, quick, easy for computer automatic processing, so these advantages have brought excellent results.
\end{abstract}

\section{Introduction}

Because gamma-ray has interaction with matter(Photoelectric effect, Compton effect, Electron pair effect, Scattering effect and so on), which makes theoretical characteristic spectrum turn into the instrument spectrum. but the gamma-ray instrument spectrum measured by NaI(TI) scintillation detector spectrometer usually consists of spectrum lines of photopeak and scattering background, if the photopeak of instrument spectrum can be analyzed accurately by means of qualitative or quantitative measures, background counting among the energy spectrum counts caused by Compton effect must be deducted effectively. At the same time, removing background counting of small angle scattering that exists in gamma-ray within sensitive volume of the detector, and restraining natural background counting, then the net count rate of measured radioactive source is to be obtained [1]. Therefore, in the analysis of radioactive gamma-ray energy spectrum, how to subtract background counting both effectively and accurately, and it is particularly important to extract parameters of characteristic peak both quickly and efficiently.

Currently, the methods for deducting background include: the trapezoidal method for smoothing spectrum data, the fitting method, the Fourier transform method, the stripping peak method, etc. Although these methods for background subtraction have been widely used with no obvious flaws, but if peak zone superimposes scattering peak and background peak during photopeak (photopeak generated by the natural radioactive source or pollutant in the laboratory environment [2]), or the characteristic X-ray peak generated by shield, the calculation result will lead to larger errors. 
However, the scattering background increases the difficulty of obtaining the response function of the detector. if statistical fluctuation at boundary of peak is larger, the impact on determining peak area and calculating the net peak area will be larger. Therefore, the paper puts forward a method of adaptive FWHM(The Full Width of peak at Half Maximum) for deducting the full spectrum background, such improved method can effectively estimate determination of peak zone, scattering peak, background subtraction, characteristic X-ray peak, etc. due to the influence on calculating net area of photopeak, it can effectively subtract the contributions of Compton scattering effect to spectra peak counts, is simple, quick, easy for computer automatic processing, and these advantages have been widely used.

\section{SNIP Algorithm Principle and Process of Background Subtraction}

Ryan and more researchers (1988) first have analyzed the principle of the SNIP(Statistics Sensitive Nonlinear Iterative Peak-Clipping Algorithm)algorithm for deducting background of the gamma energy spectrum, Morháč and more researchers (1997) have improved this algorithm [3][4]. SNIP algorithm for deducting background has better performance on Compton scattering, weakening scattering peak, background peak, and the characteristic X-ray of shield material, and has been popular. Before using the SNIP algorithm for deducting background of gamma energy spectrum, first, the use of LLS operator is to transform counting valve to each channel, the LLS operator formulation is expressed as follows [2]:

$$
v(i)=\log [\log (\sqrt{y(i)+1}+1)+1]
$$

In formula (1), $i$ is expressed as channel address, $y(i)$ is expressed as corresponding count to the $i$ channel. the logarithm operation is allowed to work on some orders of magnitudes, and square root operation enhances sensibility of weak peak choosing.

In the SNIP algorithm, through the formula(1), y(i) calculates vector $v_{1}(i), v_{2}(i) \cdots v_{m}(i)$ through iterations in sequence, in which the subscript $m$ is any given parameter. In the channel $i$, the vector value $v_{p}(i)$ of the $p$ iteration is determined by taking minimum value through comparing $v_{p-1}(i)$ with

$1 / 2\left[v_{p-1}(i+p)+v_{p-1}(i-p)\right][5][6][7]$. its mathematical expression is as follows:

$$
v_{p}(i)=\min \left\{v_{p-1}(i), \quad \frac{1}{2}\left[v_{p-1}(i+p)+v_{p-1}(i-p)\right]\right\}
$$

In formula (2), $p$ value has two changes: the first change is the beginning of iterative operation from one plus one until it is equal to given $\mathrm{m}$ value (called gradually increase method of transform window-width); the second change is the beginning of iterative operation from given $\mathrm{m}$ value minus one until it is equal to one (called gradually decrease method of conversion window-width).The location of peak zone and outside of peak zone are shown in Fig.1.
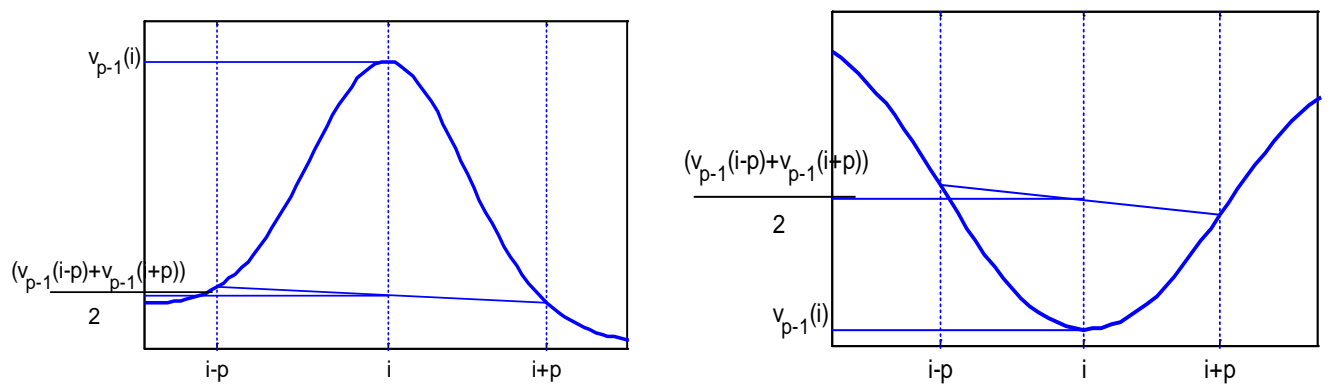

Fig.1 The location of the peak area and outside of peak area

The smoothed spectrum data is smoothed again, it will weaken the influence on statistical fluctuation nearby boundary of peak, and make the boundary of spectrum peak move to real boundary. Further smoothing makes it closer to the background in the peak zone. At this point, it is increasing the filtering window-width and accelerating the speed of filtering. When the filter window-width is $w=2 m+1$, and the $v_{\mathrm{m}}(i)$ finishes calculation, then base spectrum line of full spectrum will be obtained by inversing LLS operation. Experimental spectrum data, is what the $\Phi 75 \times 75 \mathrm{~mm} \mathrm{NaI(TI)}$ 
scintillation detector measured, the data of ${ }^{137} \mathrm{Cs},{ }^{60} \mathrm{Co},{ }^{241} \mathrm{Am}$ measured by self-developed DSP digital 2048 ch pulse amplitude analyzer, and the data of ${ }^{238} \mathrm{U}$ series and ${ }^{232} \mathrm{Th}$ series measured by 1024 ch spectrometer of CIT-3000DPP, the effects of using the traditional SNIP algorithms for deducting background of actually measured spectrum lines are shown in Fig. 2.



(1) ${ }^{241} \mathrm{Am}$ at $m=30,35,40,45$



(3) ${ }^{137} \mathrm{Cs}$ at $m=20,25,28,30$



(5) ${ }^{238} \mathrm{U}$ series at $m=20,25,30,35$

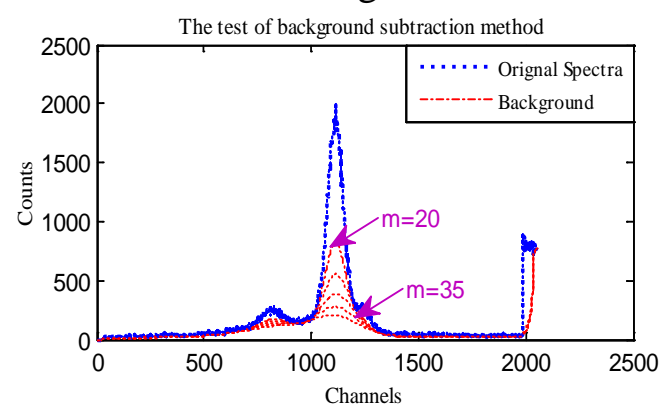

(2) ${ }^{57} \mathrm{Co}$ at $m=20,25,30,35$

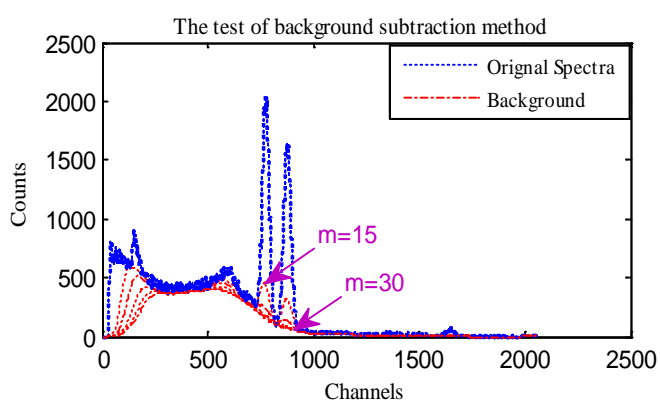

(4) ${ }^{60} \mathrm{Co}$ at $m=15,20,25,28,30$

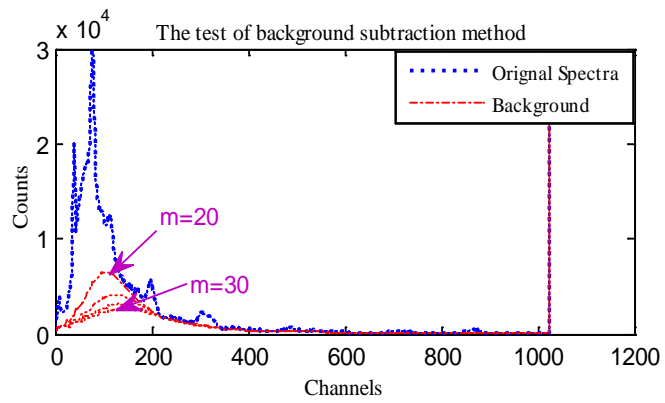

(6) ${ }^{232}$ Th series at $m=20,25,28,30$

Fig. 2 The background subtraction effect for ${ }^{241} \mathrm{Am},{ }^{57} \mathrm{Co},{ }^{137} \mathrm{Cs},{ }^{60} \mathrm{Co},{ }^{238} \mathrm{U}$ and ${ }^{232} \mathrm{Th}$ series

From figure 2, it can be known that the parameter $m$ value of transform window-width when it was linearly varying during iterations had influenced on background subtraction, $m$ value was smaller while background subtraction was more, as m value gradually increased, deducted baseline gradually smoothed. Meanwhile, if the $m$ value was determined, the filtering window-width $w$ would be determined. However, the width of spectrum peaks during full spectrum was constantly changing with different energy segment, which makes the background subtraction of gamma peaks in different regions, such as backscatter peak, Compton edge or platform and photopeak have a big deviation. Therefore, when the traditional SNIP algorithms was deducting background, parameter $\mathrm{m}$ values of conversion window-width were often determined artificially based on repeated tests, seeking adaptive matching $\mathrm{m}$ value when background need to be deducted both quickly and accurately.

\section{The Background Subtraction Method of Adaptive FWHM}

Energy resolution is the minimum variation of the difference measured by the spectrometer, The FWHM of gamma energy spectrometer is used to measure in actual measurement, $\mathrm{keV}$ is usually used as a unit. From characteristics of $\mathrm{NaI}(\mathrm{T} 1)$ detector and physical properties of forming spectrum, the response of different energy gamma photons in $\mathrm{NaI}(\mathrm{T} 1)$ detector corresponds to different FWHM of 
photoelectric peak. So-called adaptive FWHM applies optimal functions at each point to fit calibration data of FWHM. Photopeak is measured by using the gamma-ray source that is single energy among energy calibration, the FWHM and energy resolution are obtained by using fitting of the peak shape functions (such as Gaussian peak) [8][9]. Since the square of resolution and gamma-ray energy satisfies linear relationship:

$$
\delta^{2}=a+b / E
$$

Therefore, the diagram of relations $\delta^{2} \sim 1 / E$ is plotted with $\delta^{2}$ as ordinate and energy reciprocal $1 / E$ as abscissa for drawing a straight line. Value of resolution $\delta$ can be determined based on the straight line for any gamma-ray energy, so the FWHM of photopeak corresponding to its energy will be obtained. The effect of background subtraction can be valued by net count retention-rate $r(m)$ and background subtraction rate $t(m)$ of the single peak, and expressions of these two coefficients are shown as follows:

$$
\begin{aligned}
& r(m)=\frac{P N C(m)}{P N C_{0}} \times 100 \% \\
& t(m)=\frac{P N C(m)}{P T C(m)} \times 100 \%
\end{aligned}
$$

In the formula, $\mathrm{PNC}(m)$ is net counting value after background subtraction(changes with $m$ value); $\mathrm{PNC}_{0}$ is net counting value without background subtraction; PTC is total peak count after background subtraction, of which the PNC is calculated with method(TPA) of photopeak area [10]. Taking gamma energy spectrum measured by the $\mathrm{NaI}(\mathrm{Tl})$ scintillation detector into account, and its energy resolutions at low, middle, high-energy region are not same. Therefore, within the scope of whole spectrum, spectrum data of ${ }^{241} \mathrm{Am},{ }^{57} \mathrm{Co},{ }^{137} \mathrm{Cs},{ }^{60} \mathrm{Co},{ }^{238} \mathrm{U}$ series, ${ }^{232} \mathrm{Th}$ series, is selected as objects for analysis, targeted gamma-ray photopeak of several different energy section is selected and different $m$ value is taken respectively, the effect of background subtraction after observing these

\begin{tabular}{|c|c|c|c|c|c|c|c|}
\hline Nuclide & Gamma-ray Energy/keV & $\mathrm{FWHM} / \mathrm{keV}$ & Calibrations $\mathrm{keV} / \mathrm{ch}$ & $\mathrm{FWHM}(c h)$ & $m$ & $r(m) \%$ & $t(m) \%$ \\
\hline${ }^{241} \mathrm{Am}$ & 59.6 & 6.35 & 0.0465 & 136.5 & $\begin{array}{l}134 \\
136 \\
138 \\
\end{array}$ & $\begin{array}{l}100 \% \\
100 \% \\
100 \% \\
\end{array}$ & $\begin{array}{l}82.5 \% \\
86.7 \% \\
81.3 \%\end{array}$ \\
\hline${ }^{57} \mathrm{Co}$ & 122.06 & 8.83 & 0.1095 & 80.6 & $\begin{array}{l}78 \\
80 \\
82 \\
\end{array}$ & $\begin{array}{l}99.6 \% \\
99.8 \% \\
99.6 \% \\
\end{array}$ & $\begin{array}{l}89.6 \% \\
90.3 \% \\
89.4 \% \\
\end{array}$ \\
\hline${ }^{238} \mathrm{U}$ & 609.3 & 49.43 & 2.9578 & 16.7 & $\begin{array}{l}14 \\
16 \\
18\end{array}$ & $\begin{array}{l}100 \% \\
100 \% \\
100 \%\end{array}$ & $\begin{array}{l}95.4 \% \\
95.6 \% \\
93.4 \%\end{array}$ \\
\hline${ }^{137} \mathrm{Cs}$ & 661.6 & 40.86 & 0.5344 & 76.4 & $\begin{array}{l}74 \\
76 \\
78\end{array}$ & $\begin{array}{l}100 \% \\
100 \% \\
100 \%\end{array}$ & $\begin{array}{l}96.4 \% \\
96.5 \% \\
95.6 \%\end{array}$ \\
\hline${ }^{60} \mathrm{Co}$ & 1173.2 & 56.42 & 1.5138 & 37.3 & $\begin{array}{l}36 \\
38 \\
40\end{array}$ & $\begin{array}{c}99.6 \% \\
99.9 \% \\
100 \%\end{array}$ & $\begin{array}{l}95.2 \% \\
96.7 \% \\
94.4 \%\end{array}$ \\
\hline${ }^{22} \mathrm{Na}$ & 1274.5 & 56.62 & 1.5137 & 37.4 & $\begin{array}{l}36 \\
38 \\
40\end{array}$ & $\begin{array}{l}99.8 \% \\
99.9 \% \\
99.9 \%\end{array}$ & $\begin{array}{l}95.3 \% \\
95.8 \% \\
94.8 \%\end{array}$ \\
\hline${ }^{60} \mathrm{Co}$ & 1332.5 & 61.82 & 1.5138 & 40.8 & $\begin{array}{l}38 \\
40 \\
42\end{array}$ & $\begin{array}{c}99.8 \% \\
99.9 \% \\
100 \%\end{array}$ & $\begin{array}{l}99.6 \% \\
98.6 \% \\
98.6 \%\end{array}$ \\
\hline${ }^{40} \mathrm{~K}$ & 1460.85 & 77.6 & 2.9813 & 26.0 & $\begin{array}{l}24 \\
26 \\
28\end{array}$ & $\begin{array}{l}100 \% \\
100 \% \\
99.9 \%\end{array}$ & $\begin{array}{l}91.8 \% \\
92.5 \% \\
90.9 \%\end{array}$ \\
\hline${ }^{238} \mathrm{U}$ & 1764 & 87.14 & 2.9578 & 29.5 & $\begin{array}{l}28 \\
30 \\
32\end{array}$ & $\begin{array}{l}100 \% \\
100 \% \\
100 \%\end{array}$ & $\begin{array}{l}87.3 \% \\
89.1 \% \\
87.3 \%\end{array}$ \\
\hline${ }^{232} \mathrm{Th}$ & 2642 & 97.14 & 3.0438 & 31.9 & $\begin{array}{l}30 \\
32\end{array}$ & $\begin{array}{l}99.9 \% \\
100 \%\end{array}$ & $\begin{array}{l}79.9 \% \\
82.5 \%\end{array}$ \\
\hline
\end{tabular}
photopeaks are shown in Table 1.

Table 1 Impact on background subtraction of full-energy peaks with different $m$ values 
The greatest impact on the SNIP algorithm for background subtraction is value of parameter $m$, the impact on background subtraction, such as $t(m), r(m)$ and FWHM with different $m$ values in Table 1 , can be found that: (1) The difference of taking m value of Peak under different conditions may be large; (2) For most spectrum peak, when m value is matching with FWHM, the effect of background subtraction is best. Assuming that is a rule: $m$ value should match with FWHM. But characteristic of individual data is not shown in the table above, which may be caused by generated deviations for selection of peak position, determination of peak boundary, calculation of peak area or calculation of FWHM. Therefore, attempting to improve SNIP algorithm: when $m$ value automatically match with FWHM during calibrations ( $m=$ FWHM), which is also called adaptive filtering SNIP method for background subtraction. The main idea is that the full spectrum line need to be iterated until the number of iteration $m$ is equal to point of FWHM, and it should stop iterating, otherwise, it should continue to iterate until the condition is satisfied. The specific routines on MATLAB codes are shown as follows(transform window $p=m$ ):

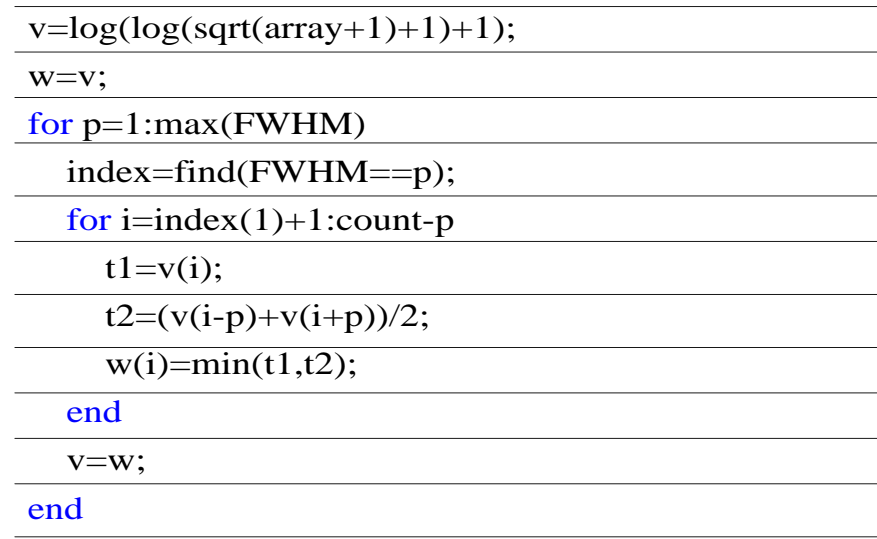

\section{Effect Analysis of Adaptive FWHM Background Subtraction}

By testing the experimental spectra data of ${ }^{241} \mathrm{Am},{ }^{57} \mathrm{Co},{ }^{137} \mathrm{Cs},{ }^{60} \mathrm{Co},{ }^{238} \mathrm{U}$ series and ${ }^{232} \mathrm{Th}$ series, the value of $m=$ FWHM is determined by adaptive filter after calibration, and the effects of background subtraction are shown in Figure 3. Red dashed line is baseline of background subtraction, since background counting is a main part of the spectrum counts caused by the Compton effects. For gamma energy spectrum that is single energy, such as ${ }^{241} \mathrm{Am},{ }^{57} \mathrm{Co},{ }^{137} \mathrm{Cs},{ }^{60} \mathrm{Co}$, it is a smoothing spectrum that changes slowly with the channel address when Compton distribution is located at the low-energy. For complex gamma energy spectrum with a variety of energy gamma peak $\left({ }^{238} \mathrm{U}\right.$ series, ${ }^{232}$ Th series ), Compton distributions of all gamma-ray peaks are superimposed together. So overall characteristics of the background will be a function that is smoothing down with energy, the effect analysis of background subtraction is known from the figure, the method also reflects the advantages on reducing scattering peak, background peak, and background characteristics X-ray of shielding materials and other aspects. 


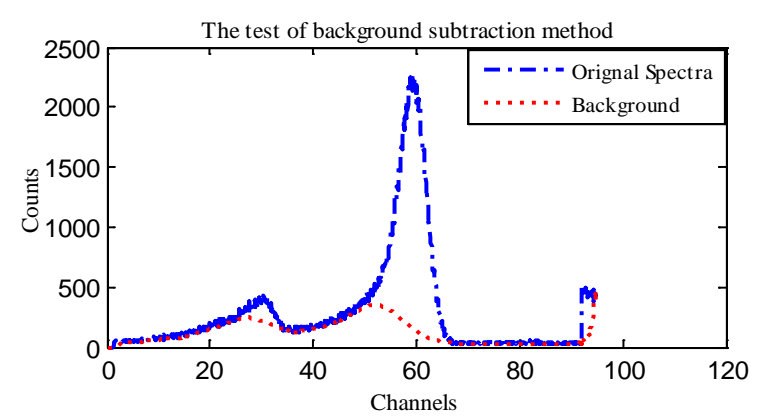

(1) ${ }^{241} \mathrm{Am}$ at $m=\mathrm{FWHM}$

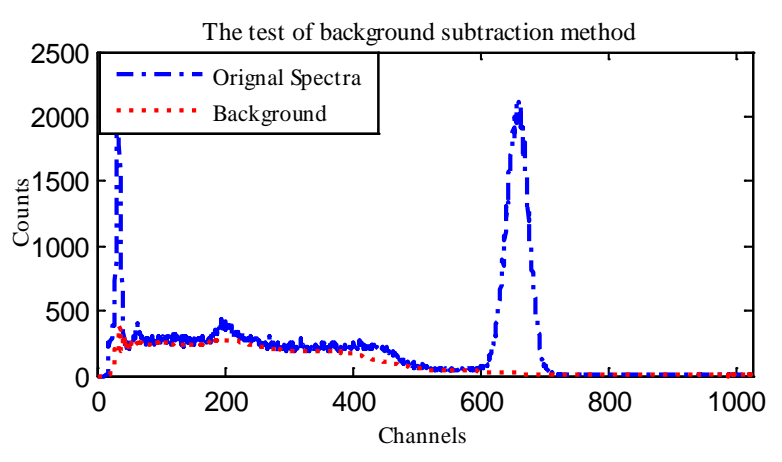

(3) ${ }^{137}$ Cs at $m=$ FWHM

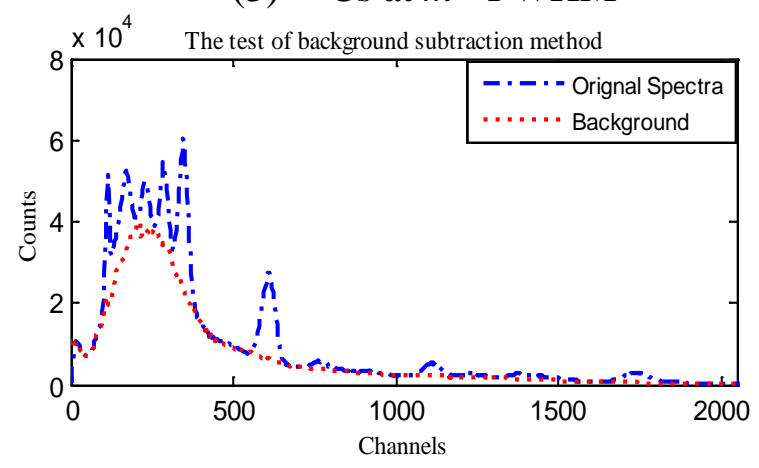

(5) ${ }^{238} \mathrm{U}$ series at $m=$ FWHM

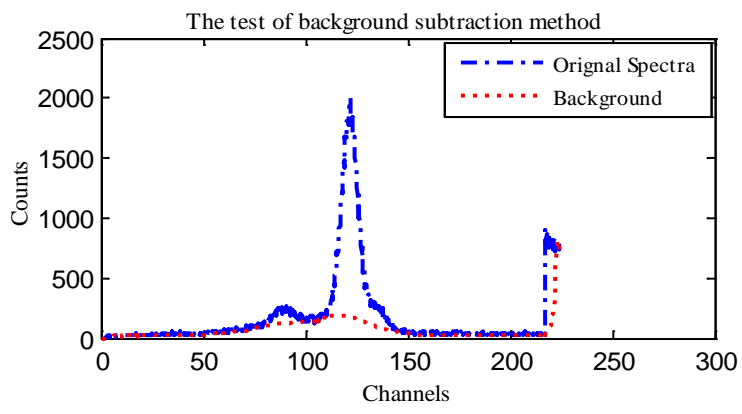

(2) ${ }^{57} \mathrm{Co}$ at $m=\mathrm{FWHM}$

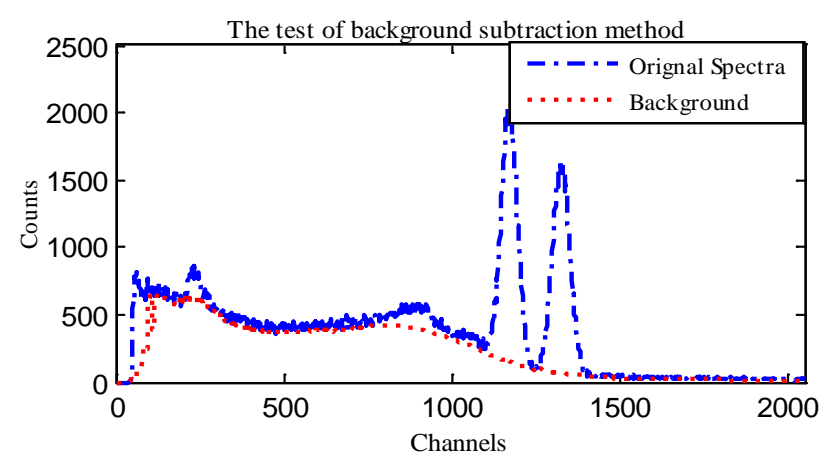

(4) ${ }^{60} \mathrm{Co}$ at $m=$ FWHM



(6) ${ }^{232}$ Th series at $m=$ FWHM

Fig. 3 The effect of background subtraction for ${ }^{241} \mathrm{Am},{ }^{57} \mathrm{Co},{ }^{137} \mathrm{Cs},{ }^{60} \mathrm{Co},{ }^{238} \mathrm{U}$ series and ${ }^{232}$ Th series $(m=$ FWHM)

Compared with figure 2, the effect of background subtraction has been significantly improved, and the accurate rule on m matching with the FWHM has been verified. Compared with primary SNIP algorithm, further improved SNIP algorithm of adaptive FWHM has obtained a good effect, and solved the question of valuing important parameter $m$, which made it have stronger practicability and better performance in automatic spectrum analysis.

\section{Summary}

Gamma-ray instrument spectrum measured by NaI(TI) scintillation detector consists of whole energy peak and scattering background. Therefore, background counting rate during measured energy counting rate must be subtracted effectively to obtain the net counting rate of radioactive source. This paper presents the method for automatically matching with the full width at half maximum (FWHM) for full spectrum deducting background-SNIP method of adaptive FWHM for background subtraction, Compared with traditional SNIP algorithm, the adaptive SNIP method improved has solved problem on the valuing parameter $m$ of transform window-width. This method is used to subtract background both effectively and accurately, and decrease the impact on determination of peak zone, scattering peak, background peak and X-ray characteristic peak. 
Meanwhile, this method has been verified with good practicability and superior reliability in the automatic spectrum analysis.

\section{Acknowledgments}

This work was supported by National Natural Science Fundation of China (No.11365001), and Educational Commission of Jiangxi Province of China (No.GJJ13464 and No.GJJ14491), and Plan of Science and Technology of Jiangxi Province (No.20141BBE50024), and Fundamental Science on Radioactive Geology and Exploration Technology Laboratory, East China Institute of Technology (No.RGET1316).

\section{References}

[1]. Jufeng Pang. Gamma energy spectrum analysis. Shanxi science and technology press,1993, p. 350-716.

[2]. Aurelian Luca, Jean Morel. Influence of the background approximation methods on the analysis of gamma-ray spectra. Applied Radiation and Isotopes. Vol.60 (2004) Issues.2-4, p. 233-237.

[3]. Miroslav Morháč, Ján Kliman, Vladislav Matoušek, et al. Background elimination methods for multidimensional coincidence $\gamma$-ray spectra. Nuclear Instruments and Methods in Physics Research Section A: Accelerators, Spectrometers, Detectors and Associated Equipment. Vol.401(1997) Issue 1, 11, p.113-132.

[4]. D.C. Radford. Background subtraction from in-beam HPGe coincidence data sets. Nuclear Instruments and Methods in Physics Research Section A: Accelerators, Spectrometers, Detectors and Associated Equipment. Vol.361(1995)Issues1-2, p.306-316.

[5]. Yi Wangming, Liu Hongzang, Tang Bin. Discussion and Application of Eliminating the background in gamma-ray Spectrum by SNIP Algorithm. Journal of East China Institute of technology.Vol.32(2009)No.3, p.245-248.

[6]. Wu Hexi, Liu Qingcheng, Yang Bo. SNIP algorithms applied to analyze gamma-ray spectra of natural radioactivity nuclides. Nuclear Techniques. Vol.33(2010) No.7, p.513-516.

[7]. Miroslav Morháč. An algorithm for determination of peak regions and baseline elimination in spectroscopic data. Nuclear Instruments and Methods in Physics Research Section A: Accelerators, Spectrometers, Detectors, Associated Equipment. Vol.600(2009)Issue.2, p.478-487.

[8]. Gordon R. Gilmore. Practical Gamma-ray Spectrometry(2nd Edition). Nuclear Training Services Ltd Warrington,UK,2008, p.131-141.

[9]. Seong-Joon Baek, Aaron Park, Jinyoung Kim, et al. A simple background elimination method for Raman spectra. Chemometrics and Intelligent Laboratory Systems.Vol.98(2009)No.1, p.24-30.

[10]. Zhou Chunlin, Xu Zhenhua , Han Feng, et al. Experimental Study of the Integral Background of a Low-background Anti-Compton HPGe $\gamma$-ray Spectrometer, Nuclear Electronics \&Detection Technology.Vol.28(2008)No.2, p.418-421. 